\title{
Increasing Levels of Minimal Residual Disease
}

National Cancer Institute

\section{Source}

National Cancer Institute. Increasing Levels of Minimal Residual Disease. NCI Thesaurus.

Code C142131.

A finding indicating an increased presence of residual disease over time. 